Abbreviated Key Title: Sch J Med Case Rep

ISSN 2347-9507 (Print) | ISSN 2347-6559 (Online)

Journal homepage: http://saspjournals.com/sjmcr

\title{
Unusual Cause of Chest Pain after Percutaneous Coronary Intervention
}

Mohd Iqbal Dar ${ }^{1 *}$, Arshed H Parry ${ }^{2}$, Amir Rashid ${ }^{3}$, Hilal A Rather ${ }^{3}$, Nisar A Tramboo ${ }^{4}$

${ }^{1}$ Senior Resident, Department of Cardiology, SKIMS Soura, J\&K, India

${ }^{2}$ Senior Resident, Department of Radiology, SKIMS Soura, J\&K, India

${ }^{3}$ Consultant, Department of Cardiology, SKIMS Soura, J\&K, India

${ }^{4} \mathrm{Head}$ of Department, Department of Cardiology, SKIMS Soura, J\&K, India

DOI: $10.36347 /$ sjmcr.2020.v08i01.026

| Received: 16.01.2020 | Accepted: 23.01.2020 | Published: 29.01.2020

*Corresponding author: Mohd Iqbal Dar

\section{Abstract}

Post procedural chest pain after percutaneous coronary intervention is a nerve wrecking symptom for both the interventionist and the patient. Prompt recognition of the underlying condition and management is critical for survival of the patient and success of the procedure. Here we describe an unusual cause of acute severe chest pain with a good prognosis on early recognition, which should be considered as one of the differential diagnosis after a coronary intervention in this scenario.

Keywords: Acute coronary syndrome, esophageal hematoma, coronary intervention, post procedure chest pain. Copyright @ 2020: This is an open-access article distributed under the terms of the Creative Commons Attribution license which permits unrestricted use, distribution, and reproduction in any medium for non-commercial use (NonCommercial, or CC-BY-NC) provided the original author and source are credited.

\section{INTRODUCTION}

Acute chest pain in the immediate post procedure period after a coronary intervention usually adversely effects the procedural and patient outcomes. Out of the most common and catastrophic cause's Esophageal hematoma is a very uncommon contributor. It has a good prognosis and is usually managed conservatively but its diagnosis requires high index of suspicion and careful dissection clinical signs and symptoms.

\section{CASE RePORT}

A 60 years old male hypertensive and diabetic presented with complaints of chest pain and evaluation in emergency department showed patient having acute coronary syndrome with inferior wall myocardial infarction and patient was thrombolysed with reteplase successfully. Patient was subsequently taken for coronary angiography and PCI to right coronary artery was done.

Next day the patient started with acute sever chest pain radiating to shoulders and back and was associate with diaphoresis. Serial ECGs were done which showed no fresh changes and troponin levels were repeated which did not show any peaking than the levels recorded earlier. Patient was given analgesics but patient continued to be symptomatic and developed symptom of dysphagia. In view of the clinical scenario it was decided to do a CT angiography to rule out acute aortic syndrome. The CT showed normal aorta but note was made of a $3.7 \times 2.2 \times 2.3 \mathrm{~cm}$ non-enhancing intramural area at the lower esophagus which is hyperdense on non-contrast study suggestive of intramural esophageal wall hematoma figure 01 .

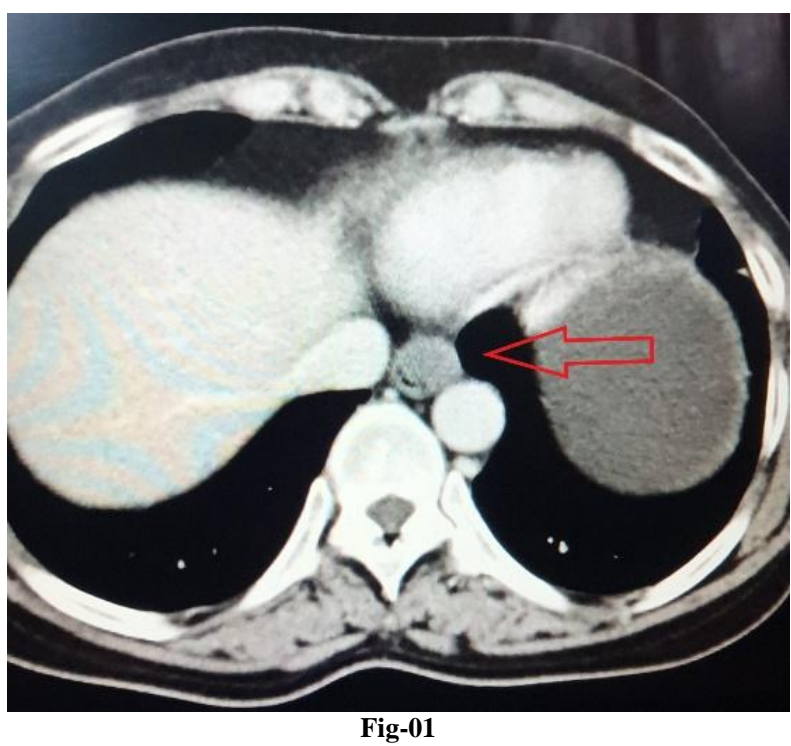

Esophageal gastro duodenal (EGD) endoscopy was done subsequently which showed bluish discoloration with a long bulge in the lower esophagus suggestive of intramural esophageal hematoma figure 02 . 


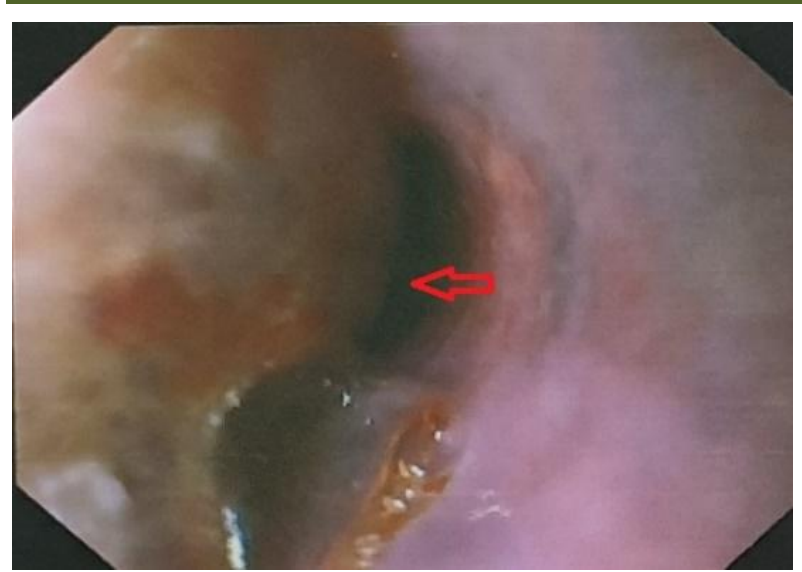

Fig-02

Patient was managed conservatively. Patient was asked to avoid oral intake and was given intravenous hydration and some analgesics and monitored closely for symptoms of upper gastrointestinal bleed. Symptoms began to improve from second day and orals were started from third day and patient was discharged in stable condition.

\section{DISCUSSION}

Esophageal hematoma is caused due to separation of esophageal lining from the muscular layer with accumulation of blood between the two layers [1]. It is usually a disease of elderly and fragile people on anticoagulants, occurring spontaneously. It can also occur after upper GI endoscopy, endotracheal intubation and foreign body ingestion. It is also known as esophageal apoplexy, esophageal dissection and intramural dissection of esophagus and usually involves the distal part of esophagus [2]. Esophageal hematoma has to be differentiated from other severe acute esophageal syndromes of Berhaave's syndrome and Mallory-Weiss syndrome. Berhaave's syndrome is caused by rupture of esophagus in to mediastinum leading to subcutaneous emphysema and mediastinal infection leading to sepsis. Mallory -Weiss syndrome is caused due to tear in the esophageal mucosa leading to upper GI bleed following recurrent bouts of sever retching and vomiting [3].

Esophageal hematoma presents with acute retrosternal pain radiating to back creating resemblance with clinical presentation of aortic dissection. This is followed by dysphagia. Dysphagia is usually reported late in post coronary intervention period as the patients are usually advised to not to take orally in this scenario [4]. Upper GI bleed in the form of hematemesis can occur due to rupture of hematoma. The classical triad of chest pain, dysphagia and hematemesis is presentation in only one third of the patients [5].

During coronary intervention the role of antiplatelets and anticoagulants in development of esophageal hematoma is unclear and inconclusive. Anticoagulants and antiplatelets are thought to not cause but aggravate the formation esophageal hematoma. Patients developing esophageal hematoma are likely to have predisposition for developing the condition $[6,7]$. Our patient received both antiplatelets and anticoagulants and both may have played a role in developing the hematoma. In view of the clinical presentation aortic dissection is usually considered as first differential and a computer tomography (CT) is invariably performed. Fortunately CT has a high sensitivity for detection of esophageal hematoma and may be bring the first clue towards making this diagnosis [8, 9]. Esophageal endoscopy can directly visually the hematoma, confirm diagnosis and institute local therapy if required. Other modalities of diagnosis include endoscopic ultrasound and magnetic resonance imaging $[10,11]$.

Management of esophageal hematoma is mostly conservative. It includes restricting oral, IV fluids and analgesics. Follow up scans usually show resolution of hematoma completely in 3 to 4 weeks [1214].

\section{CONCLUSION}

Esophageal hematoma can clinically mimic as a catastrophic chest pain after coronary intervention and is usually not considered in view of low awareness of the treating physicians. This can lead to misdiagnosis causing unnecessary morbidity and mortality in a relatively benign disease.

\section{REFERENCES}

1. Chiu YH, Chen JD, Hsu CY, How CK, Yen DH, Huang CI. Spontaneous esophageal injury: esophageal intramural hematoma. J Chin Med Assoc. 2009;72:498-500.

2. Clark W, Cook IJ. Spontaneous intramural hematoma of the oesophagus: radiologic recognition. Australas Radiol. 1996;40:269- 272.

3. Tay YK, Tay JY, Dandie L, Gribbin J. Intramural hematoma of the esophagus mimicking hemorrhaging esophageal cancer. Annals of Gastroenterology: Quarterly Publication of the Hellenic Society of Gastroenterology. 2013;26(1):74.

4. Abdi S, Baianati MR, Momtahen M, Mohebbi B. Intramural Esophageal Dissection: A Rare Cause of Acute Chest Pain after Percutaneous Coronary Intervention. The Journal of Tehran University Heart Center. 2019 Sep 7.

5. Geller A, Gostout CJ. Esophagogastric hematoma mimicking a malignant neoplasm: clinical manifestations, diagnosis, and treatment. Mayo Clin Proc. 1998;73:342-345

6. Meulman N, Evans J, Watson A. Spontaneous intramural haematoma of the oesophagus: a report of three cases and review of the literature. Aust N Z J Surg. 1994; 64:190-193. 
7. Van Laethem JL, Devière J, Cremer M. Serial endoscopic findings of spontaneous intramural hematoma of the esophagus. Endoscopy. 1997; 29:44-46.

8. Ouatu-Lascar R, Bharadhwaj G, Triadafilopoulos G. Endoscopic appearance of esophageal hematomas. World J Gastroenterol. 2000; 6:307309.

9. Abbey P, Sharma R, Garg PK. Spontaneous intramural haematoma of the oesophagus: complete resolution on follow-up magnetic resonance imaging. Singapore Med J. 2009; 50:e318-320.

10. Meininger $M$, Bains $M$, Yusuf $S$, Gerdes $H$. Esophageal intramural hematoma: a painful condition that may mimic an esophageal mass. Gastrointest Endosc. 2002;56:767-770.
11. Kamphuis AG, Baur CH, Freling NJ. Intramural hematoma of the esophagus: appearance on magnetic resonance imaging. Magn Reson Imaging. 1995; 13:1037-1042.

12. Meulman N, Evans J, Watson A. Spontaneous intramural haematoma of the oesophagus: a report of three cases and review of the literature. Aust N Z J Surg. 1994; 64:190-193.

13. Ivekovic H, Ivekovic MP. Intramural esophageal hematoma: an unusual complication of endotracheal intubation. J Emerg Med. 2012 (in press)

14. Kerr WF. Spontaneous intramural rupture and intramural haematoma of the oesophagus. Thorax. $1980 ; 35: 890-897$. 